\title{
Terapia láser en afectación ocular tras el diagnóstico de incontinencia pigmenti en una niña
}

\author{
Laser therapy in ocular involvement after diagnosis of incontinentia pigmenti \\ in a child
}

\author{
Lic. Felisa Vázquez Gómez ${ }^{a}$ Lic. Laura Díaz Ruiza, Lic. Elena Bergón Sendín ${ }^{b}$ Dra. Ana Barceló Mendiguchía \\ y Dra. Pilar Tejada Palacios ${ }^{c}$
}

\section{RESUMEN}

La incontinencia pigmenti es un trastorno neurocutáneo raro, con una frecuencia de 1 en 40000 recién nacidos, de etiología genética asociada a mutaciones en el gen $I K B K G$, localizado en Xq28, con herencia dominante ligada al X. Tiene una presentación clínica de manifestaciones muy variables detectadas desde la etapa neonatal y puede asociar afectación cutánea, dental, ocular y neurológica, y cada una de estas con un diagnóstico diferencial distinto.

Se presenta a una paciente pediátrica con diagnóstico de incontinencia pigmenti a la semana de vida. En la evaluación oftalmológica inicial, se observaron lesiones vasculares retinianas. Se decidió el tratamiento con láser, con buenos resultados, y se consiguió estabilizar la visión.

Palabras clave: incontinencia pigmentaria, manifestaciones oculares, lesiones del sistema vascular, terapia por láser, pediatría.

\begin{abstract}
Incontinentia pigmenti is a rare neurocutaneous disorder with a frequency of 1 in 40,000 newborn; it is associated with mutations in IKBKG gene in $\mathrm{Xq} 28$, inherited as an X-linked dominant trait. Clinical manifestations detected since the newborn period are highly variable, with skin, teeth, eyes, and nervous system manifestations, and each with a characteristic differential diagnosis.

We present a pediatric patient diagnosed with incontinentia pigmenti at the first week of life. In the initial ophthalmologic evaluation, retinal vascular lesions were observed. The outcomes of laser treatment of the ischemic peripheral retina were good and resulted in stability of vision.

Key words: incontinentia pigmenti, eye manifestations, vascular system injuries, laser therapy, pediatrics.
\end{abstract}

a. Servicio de Pediatría.

b. Unidad de Neonatología,.

c. Sección de Oftalmología Pediátrica.

Hospital Universitario 12 de Octubre, Madrid, España.

Correspondencia:

Lic. Felisa Vázquez Gómez: felisa.vazquezg@gmail.com

Financiamiento: Ninguno.

Conflicto de intereses: Ninguno que declarar.

Recibido: 4-2-2018

Aceptado: 6-12-2018 http: / / dx.doi.org/10.5546/ aap.2019.e252

Cómo citar: Vázquez Gómez F, Díaz Ruiz L, Bergón Sendín E, Barceló Mendiguchía A, Tejada Palacios P. Terapia láser en afectación ocular tras el diagnóstico de incontinencia pigmenti en una niña. Arch Argent Pediatr 2019;117(3):e252-e256.

\section{INTRODUCCIÓN}

La incontinencia pigmenti ${ }^{1}$ (IP) o síndrome de Bloch-Sulzberger es una genodermatosis rara, vinculada al cromosoma $X$, de carácter dominante, que afecta a tejidos ectodérmicos y mesodérmicos, como la piel, los ojos, los dientes y el sistema nervioso central. La incidencia estimada es de 1 cada 40000 niños. Las mutaciones del gen IKBKG localizado en la porción q28 del cromosoma $X$ causan la expresión de la enfermedad; una única mutación es la responsable de alrededor de un $80 \%$ de los casos. ${ }^{2}$ Un $95 \%$ de los pacientes afectos serán mujeres, pero, en ocasiones, puede ocurrir en varones que presenten un mosaicismo o un cariotipo XXY. ${ }^{3}$

Clínicamente, el defecto cutáneo está siempre presente y se considera el principal criterio diagnóstico de acuerdo con Landy y Donnai. ${ }^{4}$ Evoluciona en 4 etapas: vesiculosa, verrugosa, hiperpigmentada e hipopigmentada, la cual suele perdurar toda la vida.

Aun así, la gravedad de la enfermedad está relacionada con la presencia de trastornos neurológicos y/u oculares, que están presentes, respectivamente, en un $30 \%$ y en un $35-70 \%$ de los pacientes. ${ }^{1}$

\section{CASO CLÍNICO}

Se presenta a una recién nacida a término de bajo peso para la edad gestacional con embarazo normal y controlado. Nacida por parto eutócico a las 40 semanas de gestación sin precisar reanimación, con un puntaje de Apgar de 9/10. Presentó un peso de 2470 gramos (< percentil 10), talla de $45 \mathrm{~cm}$ y perímetro cefálico 
de 31,5 cm, sin llegar a identificar la etiología del bajo peso. A nivel familiar, se destacaba la madre con diagnóstico de distrofia corneal endotelial hereditaria y el padre con diabetes mellitus tipo I y un hermano de 4 años, varón, sano.

En la exploración durante el primer día de vida, la paciente presentó eritema de distribución lineal que seguía las líneas de Blaschko en ambos miembros inferiores y superiores, con lesiones vesiculosas en el muslo derecho, en el tercio distal de la pierna derecha, en el tercio distal de la pierna izquierda y, más evidentes, en el miembro superior derecho. Eritema difuso en el tronco y en las nalgas, sin otros hallazgos de interés (Figura 1).

Entre la etiología para descartar, se encontraba la infección por virus herpes simplex, infección local por Staphylococcus aureus (impétigo) y eritema tóxico del recién nacido, aunque ni la evolución de las lesiones ni la historia clínica resultaban compatibles con ellas. Se realizó la interconsulta con el Servicio de

Figura 1. Lesiones iniciales cutáneas a las 24 horas de vida (miembro inferior derecho)

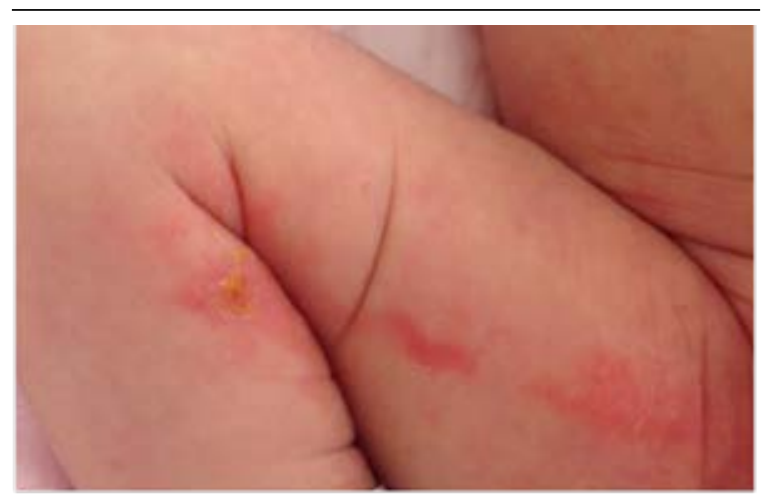

Dermatología y se decidió biopsiar las lesiones para su estudio histológico, con la sospecha de IP fase I. Se completó la evaluación con hemograma y bioquímica, así como con ecografía transfontanelar, y no se evidenciaron alteraciones, salvo una leve eosinofilia en la sangre periférica (hemoglobina: 18,9 g/ dl; hematocrito: 57,8\%; volumen corpuscular medio (VCM): 108,2 fl; plaquetas: $286000 / \mu \mathrm{l}$; leucocitos: $8900 / \mu \mathrm{l}$ (neutrófilos: $2800 / \mu \mathrm{l}$-el 30,7 \%-; linfocitos: 4000/ $\mu \mathrm{l}$-el 44,6\%-; monocitos: $1400 / \mu \mathrm{l}-\mathrm{el} 15,8 \%$-; eosinófilos: $700 / \mu \mathrm{l}-\mathrm{el}$ 8,3\%-; basófilos: $0 / \mu \mathrm{l}$ -el $0 \%-)$.

Días después, se recibió el informe anatomopatológico, en el que se observó piel que presentaba acantosis, espongiosis y abundantes eosinófilos intraepidérmicos entre los queratinocitos. En la dermis papilar, se observó un infiltrado linfocitario perivascular con aislados eosinófilos. En la zona periférica de la epidermis, se reconocieron también queratinocitos necróticos (disqueratosis) junto con eosinófilos.

Se confirmó, de este modo, una dermatosis eosinofílica compatible con IP.

Debido a las manifestaciones que podía asociar esta enfermedad, se solicitó una resonancia magnética (RM) cerebral a las dos semanas de vida, en la que se observaron lesiones difusas en sustancia blanca (Figura 2), así como la evaluación por Oftalmología, que visualizó, en el ojo izquierdo, la amputación periférica de vasos con neoformación en ovillo temporal y la dilatación de vasos retinianos a nivel ecuatorial, sin exudados ni hemorragias ni desprendimiento de retina (Figura 3) y, en el ojo derecho, la amputación periférica de vasos, sin neovasos.

FIGURA 2. Imágenes de la resonancia magnética craneal a los 14 días de vida, en la que se identificaron lesiones en la sustancia blanca inespecíficas sin repercusión clínica en la evolución
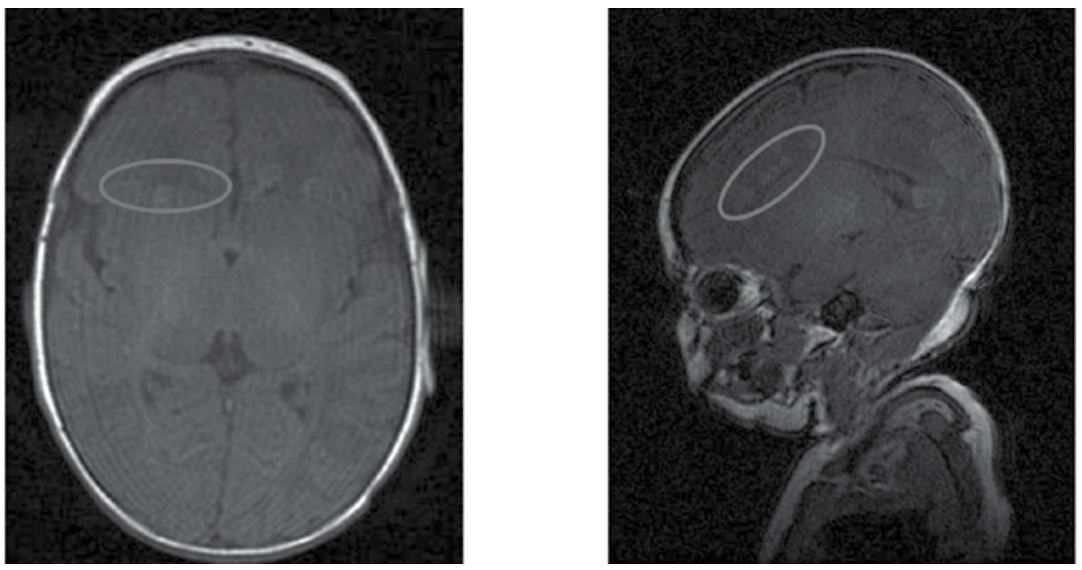
Ante estos hallazgos, se planteó el seguimiento estrecho por parte de Dermatología, Neurología y Oftalmología Pediátrica, que propusieron a la paciente para terapia con láser diodo en el ojo izquierdo.

La intervención se realizó a los 2 meses de vida, sin incidencias, y evolucionó de forma muy favorable. A los 15 días del procedimiento, no se observaban neovasos en el ojo izquierdo (Figura 4), y las zonas cicatriciales mantenían buen aspecto.

En nuestra paciente, se realizó un estudio genético, que mostró la deleción del gen IKBKG, mutación característica de este cuadro.
A nivel cutáneo, la paciente presentaba, a los 15 días de vida, lesiones costrosas, junto con un eritema muy difuso que seguía las líneas de Blaschko en la cara interna de ambas piernas, con resolución al mes, sin necesidad de iniciar el tratamiento dirigido. A los 6 meses de edad, se observaban placas de hiperpigmentación residuales de morfología lineal que seguían las líneas de Blaschko, que desaparecieron al año de vida y no volvieron a aparecer hasta el momento actual.

Transcurridos los 36 meses desde el nacimiento, la paciente presentaba desarrollo psicomotor normal y continuaba el seguimiento

FIGURA 3. Fondos de ojo (derecho e izquierdo) al nacer con lesiones establecidas
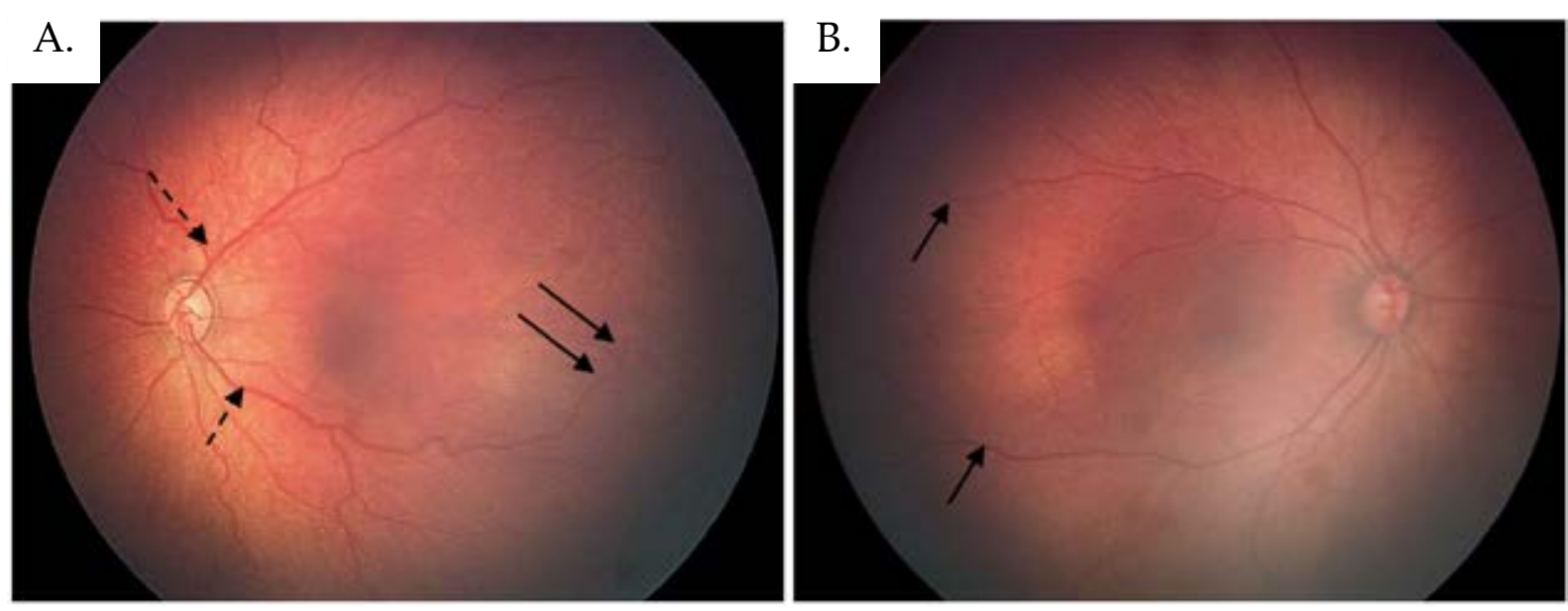

A) Fondo de ojo inicial del ojo izquierdo: se visualiza la amputación periférica de vasos con neoformación en ovillo temporal (flechas continuas), dilatación de vasos retinianos a nivel ecuatorial (flechas discontinuas).

B) Fondo de ojo inicial del ojo derecho: amputación periférica de vasos, sin observarse nuevas formaciones vasculares (flechas continuas).

Figura 4. Evolución del fondo de ojo tras la intervención con láser

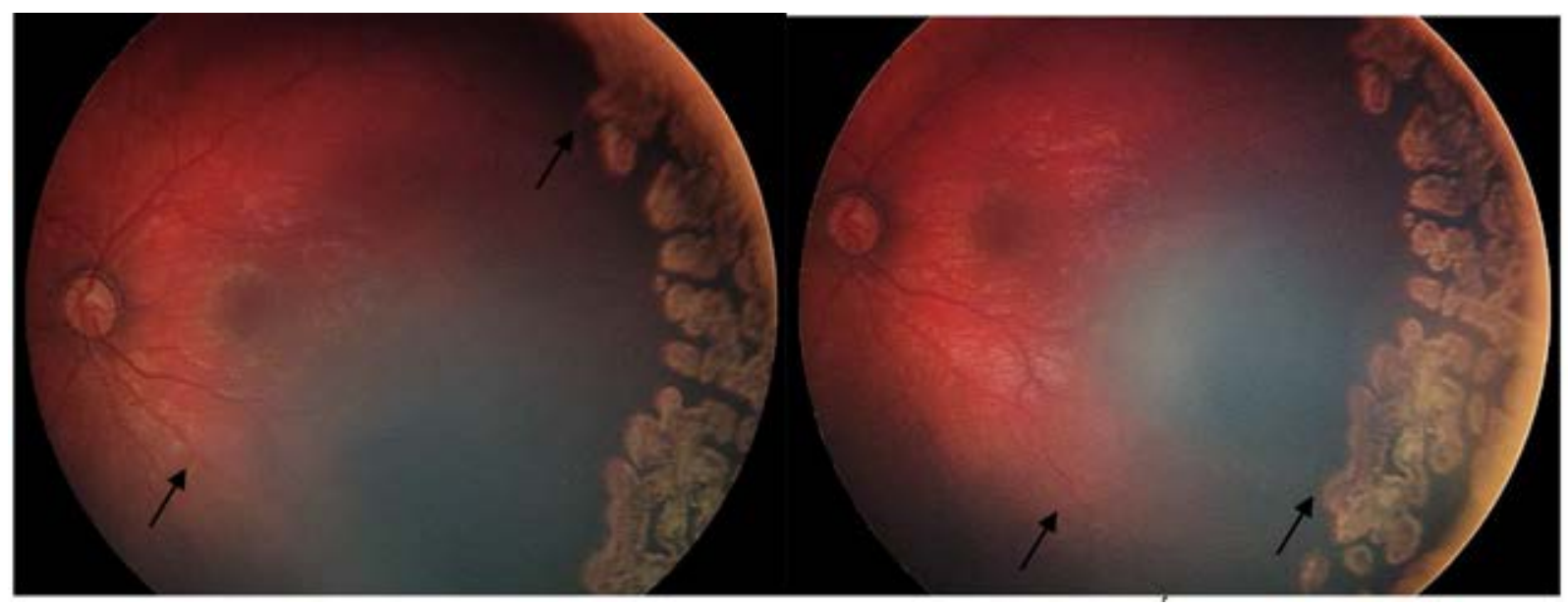

Lesiones cicatriciales de buen aspecto, sin nuevas formaciones vasculares (flechas continuas). 
con Neurología Pediátrica. En la RM cerebral de control al año y a los dos años de vida, se identificaron lesiones de sustancia blanca similares a la RM inicial, que no presentaban correlación clínica; no asociaba dismorfias ni alteraciones de la dentición.

Mantenía controles periódicos en Oftalmología, sin haberse observado nuevas formaciones vasculares. Portadora de lentes correctoras por astigmatismo e hipermetropía, conservaba buena agudeza visual bilateral, con leve microftalmia del ojo izquierdo.

\section{COMENTARIO}

Las manifestaciones oculares en la IP no son las más comunes, pero, a menudo, asocian alta morbilidad. ${ }^{1}$ A diferencia de los síntomas dermatológicos, que se atenúan con los años, la afectación ocular persiste a lo largo de la vida y afecta la calidad de esta.

Las primeras señales cutáneas consisten en erupciones lineales con vesiculación y distribución preferencial en el tronco y en los miembros. ${ }^{5,6}$ Algunas semanas después, aparecen lesiones verrugosas hiperqueratóticas, distribuidas, preferencialmente, en los miembros y en el cuero cabelludo. Luego (12-16 semanas), se hacen visibles las zonas de hiperpigmentación, la fase más característica, que afecta, por lo general, el tronco, las axilas, las regiones inguinales y las regiones mamilares; progresivamente, se van atenuando y pueden desaparecer en la pubertad $\mathrm{y}$ en la fase adulta o incluso antes.

Los tres estadios pueden coexistir, pero también puede ausentarse alguno. La cuarta fase es una manifestación tardía basada en lesiones hipopigmentadas, sobre todo, en los miembros inferiores.

Ante las lesiones iniciales, es esencial excluir dermatosis de mayor morbilidad, tales como el impétigo, las lesiones herpéticas, las dermatosis ampollosas congénitas neonatales y las enfermedades autoinmunes. ${ }^{1,5,6}$ No requieren tratamiento específico, pero sí controles cutáneos periódicos.

Además, los trastornos del sistema nervioso central constituyen una importante alteración en la calidad de vida en los pacientes con IP, y la prevalencia, aproximadamente, es del $30 \%{ }^{7}$ Las manifestaciones neurológicas descritas son muy variadas: convulsiones o status mantenido, retraso psicomotor, deficiencia intelectual, hemiplejía, epilepsia, ataxia cerebelosa, microcefalia, encefalopatía neonatal, encefalitis y lesiones vasculares.

En los pacientes con IP, es esencial realizar una prueba de imagen del sistema nervioso central al conocer o sospechar el diagnóstico. Las lesiones más frecuentemente encontradas son infartos, zonas atróficas y lesiones del cuerpo calloso. ${ }^{8}$ Dependiendo de la clínica y de las lesiones, precisarán diversos tratamientos. Es esencial el seguimiento de los pacientes para identificar un adecuado desarrollo psicomotor o variables de este.

A nivel oftalmológico, las lesiones más características y más graves son las retinianas, que afectan a la vascularización en el desarrollo durante los primeros meses de vida y al epitelio pigmentario retiniano. ${ }^{9}$ La historia natural y la patogénesis oftálmica de este síndrome son poco conocidas, aunque el proceso isquémico y la subsiguiente respuesta angiogénica son ligeramente similares a la retinopatía del prematuro. La prevalencia de ceguera unilateral o bilateral se ha descrito entre el $7 \%$ y el $23 \%$ de los afectos. ${ }^{9}$

Los cambios observados en la retina suelen ser secundarios a la isquemia y a la posterior evolución: proliferación de nuevos vasos sanguíneos, exudación, gliosis prerretiniana y tracción/desprendimiento de retina. El proceso puede ser autolimitado, detenerse en cualquier etapa y dejar secuelas, como áreas avasculares, tortuosidad vascular, exudados, hemorragia vítrea, fibrosis prerretinal, cambios en la pigmentación del epitelio pigmentario retiniano, masa retrolental y desprendimiento de retina.

Como tratamiento ante dichos eventos, se puede emplear la fotocoagulación láser, la cual se ha descrito de gran utilidad ante la acción proliferativa vascular retiniana, como el caso de nuestra paciente, que permite estabilizar la visión y evitar la disminución de la calidad de vida. ${ }^{10,11}$

Entre los hallazgos no retinianos, los más comunes son las cataratas y el estrabismo. ${ }^{9}$ Otros cambios reportados han sido ptosis, microftalmia, esclerótica azul, pigmentación conjuntival, alteraciones corneales, hipoplasia del iris, uveítis, atrofia del globo ocular, nistagmo y miopía.

Debido a la gravedad de las lesiones, un programa de cribado ocular y seguimiento es imprescindible para los niños con diagnóstico de IP para permitir la detección temprana de alteraciones y la intervención terapéutica, ya que, si no son tratados, estos defectos pueden progresar a una ceguera irreversible. ${ }^{11}$ 
En definitiva, la IP en una enfermedad genética potencialmente grave que obliga a un manejo multidisciplinar del paciente afecto desde los primeros días de vida.

\section{REFERENCIAS}

1. Poziomczyk CS, Recuero JK, Bringhenti L, Maria FD, et al. Incontinentia pigmenti. An Bras Dermatol. 2014; 89(1):26-36.

2. Conte MI, Pescatore A, Paciolla M, Esposito E, et al. Insight into IKBKG / NEMO locus: report of new mutations and complex genomic rearrangements leading to incontinentia pigmenti disease. Hum Mutat. 2014; 35(2):165-77.

3. Alabdullatif Z, Coulombe J, Steffann J, Bodemer C, et al. Postzygotic mosaicism and incontinentia pigmenti in male patients: molecular diagnosis yield. Br J Dermatol. 2018; 178(4):e261-2

4. Landy SJ, Donnai D. Incontinentia pigmenti (BlochSulzberger syndrome). J Med Genet. 1993; 30(1):53-9.

5. Fusco F, Paciolla M, Conte MI, Pescatore A, et al. Incontinentia pigmenti: report on data from 2000 to 2013.
Orphanet J Rare Dis. 2014; 9:93.

6. GianfaldoniS, Tchernev G, Wollina U, Lotti T. Incontinentia Pigmenti: A Case Report of a Complex Systemic Disease. Open Access Maced J Med Sci. 2017; 5(4):501-5.

7. SoltirovskaSalamon A, LichtenbeltK, Cowan FM, Casaer A, et al. Clinical presentation and spectrum of neuroimaging findings in newborn infants with incontinentia pigmenti. Dev Med Child Neurol. 2016; 58(10):1076-84.

8. Minić S, Trpinac D, Obradović M. Systematic review of central nervous system anomalies in incontinentia pigmenti. Orphanet J Rare Dis. 2013; 8:25.

9. O'Doherty M, McCreery K, Green AJ, TuwirI, et al. Incontinentia pigmenti: ophthalmological observation of a series of cases and review of the literature. Br J Ophthalmol. 2011; 95(1):11-6.

10. Nguyen JK, Brady-Mccreery KM. Laser photocoagulation in preproliferative retinopathy of incontinentia pigmenti. J AAPOS. 2001; 5(4):258-9.

11. Batioglu F, Ozmert E. Early Indirect Laser Photocoagulation to induce regression of retinal vascular abnormalities in incontinentia pigmenti. Acta Ophthalmol. 2010; 88(2):267-8. 